\title{
Cutaneous Schwannoma Presenting as a Parotid Tumour - A Case Report and Literature Review
}

\author{
Jugmohansingh $G^{1} \mid$ Islam $S^{2} \mid$ Clarke ${ }^{1} \mid$ Mohip $S^{1} \mid$ Christopher $M^{1} \mid$ Acco O ${ }^{2}$
}

${ }^{1}$ Department of Otolaryngology, San Fernando General Hospital, Trinidad and Tobago

${ }^{2}$ Department of General Surgery, San Fernando General Hospital, Trinidad and Tobago

\begin{abstract}
Schwannomas are nerve sheath tumours that can occur at any point along the course of spinal nerves, peripheral nerves and most cranial nerves. The cutaneous schwannoma is uncommon and accounts for $5 \%$ of schwannomas. They are slow growing, mostly benign tumours that develop within the dermis of the skin or subcutaneous tissues. Cutaneous schwannomas can be present in the head and neck but growth in the region of parotid gland is not usual and can lead to diagnostic confusion as the parotid gland can also develop schwannomas. We now present the case of a 19 year-old female who presented with a right sided facial mass in the parotid region. Initial tru cut biopsy confirmed the presence of Antoni A and Antoni B type areas with immunochemistry staining positive for S-100. A diagnosis of a parotid schwannoma was made. However, intra-operatively, the mass was found to lie above the superficial musculo-aponeurotic system and a revised diagnosis of a cutaneous schwannoma was made. Observation is the mainstay of treatment. However, surgical resection is performed if the patient has pain, structural dysfunction, cosmetic concerns of if the tumour is malignant. Radiotherapy and chemotherapy have limited roles in management.
\end{abstract}

Keywords: Head and neck schwannoma, cutaneous schwannoma, benign peripheral nerve sheath tumour

Copyright : (C) 2021 The Authors. Published by Publisher. This is an open access article under the CC BY-NC-ND license (https://creativecommons.org/licenses/by-nc-nd/4.0/).

\section{1 | INTRODUCTION}

$\mathrm{S}$ chwannomas are tumours that originate from cells found within neural sheaths called schwann cells. These neoplasms can occur at any point along the course of spinal nerves, peripheral nerves and most cranial nerves . $(1,2)$ The schwannona develops within one fascicle of the nerve sheath which then grows to displace the rest of structures within that nerve bundle. Five variants 


\section{INNOVATIVE JOURNAL}

have been described: cellular, plexiform, epithelioid, melanotic, and ancient forms. (3)Schwannomatosis which is the occurrence of multiple schwannomas can occur in $2 \%$ of patients. (4) Schwannomas can also be associated with other medical conditions such as neurofibromatosis type 2, Carney complex and intracranial tumours. (4) In the head and neck, tumours frequently occur within the cranium, spine or within deep tissues. (2) Extracranial schwannomas occur in $25-45 \%$ of cases. (5) However, cutaneous schwannomas are uncommon and account for only $5 \%$ of cases. (4)

Here, we present the case of a 19 year-old female who presented with a right facial mass in the parotid region. Initial trucut biopsy confirmed the presence of a schwannoma and a diagnosis of a parotid schwannoma was made. However, intra-operatively, the mass was found to lie above the superficial musculoaponeurotic system (SMAS). A revised diagnosis of a cutaneous schwannoma of the face was then made. The management of a cutaneous schwanoma of the head and neck will then be discussed.

Case Report:A 19-year-old female of East Indian descent developed a painless, slow-growing mass over the right jaw which was present for a period of 2 years. She had initially consulted a dermatologist who ordered an ultrasound scan. This had reported a $2.7 \times 2.4 \times 1.7 \mathrm{~cm}$ mass in the right parotid region. A truct biopsy of the mass was then performed and this identified cells with elongated nuclei arranged in a palisading pattern (Antoni A type) on a background of fibrillary stroma suggestive of a spindle cell lesion favouring a schwannoma. Immunohistochemistry was S-100 positive confirming the diagnosis. A parotid schwannoma was diagnosed and the patient was subsequently referred to the Otolaryngology Department of the San Fernando General Hospital.

A facial swelling was noted over the ramus of the right mandible in the region of the parotid gland.

Supplementary information The online version of this article (10.52845/IJMHS/2021-11-09-21) contains supplementary material, which is available to autho-rized users.

San Fernando General Hospital, Trinidad and Tobago
A small scar was also seen at the area where the trucut biopsy was performed. The mass measured 3 $\mathrm{x} 2 \mathrm{~cm}$ in size and was firm but non-tender. It was fixed to the skin at the point where the trucut biopsy was performed but was not fixed to the underlying deep structures. It was mobile in horizontal as well as vertical planes and was still mobile after the patient clenched their teeth. This mobility was not in keeping with that of a parotid lesion. The patient was then counselled on surgical excision of a facial mass but was also counselled on facial nerve dysfunction in case the tumour did originate in the superficial parotid lobe.

At surgery, the area of skin to be excised was identified by an inner elliptical marker. An outline of the mass itself was indicated by a marker along the outer margin Figure 1. A modified Blair incision was made and the skin flap was raised over the SMAS layer. During this process, the lesion was seen under the facial skin within the subcutaneous tissue Figure 2. It was attached to the skin at the biopsy site (fibrosis and scar tissue secondary to the trucut biopsy) and this area of skin was also removed.. The entire tumour was dissected above the SMAS layer Figure 3 . The parotid gland was not exposed during the resection of the lesion at any point. The cervical aspect of the incision did not have to be dissected thus limiting the extent of the scar. The wound was repaired in layers using absorbable sutures.

The histology confirmed the diagnosis of a schwannomaFigure 4 . This as well as the superficial location of the tumour suggested an origin from the neural sheath of a peripheral cutaneous nerve. The diagnosis of a cutaneous schwannoma of the face was made. One week later the patient was seen in the clinic. A general examination did not identify any café aulait spots or other abnormal swellings on her body. The sutures were removed and the patient had an uneventful recovery. Six months later, there have been no signs of regrowth.

\section{2 | DISCUSSION:}

Schwann cells provide insulation and improve nerve conduction within cranial, spinal and peripheral 


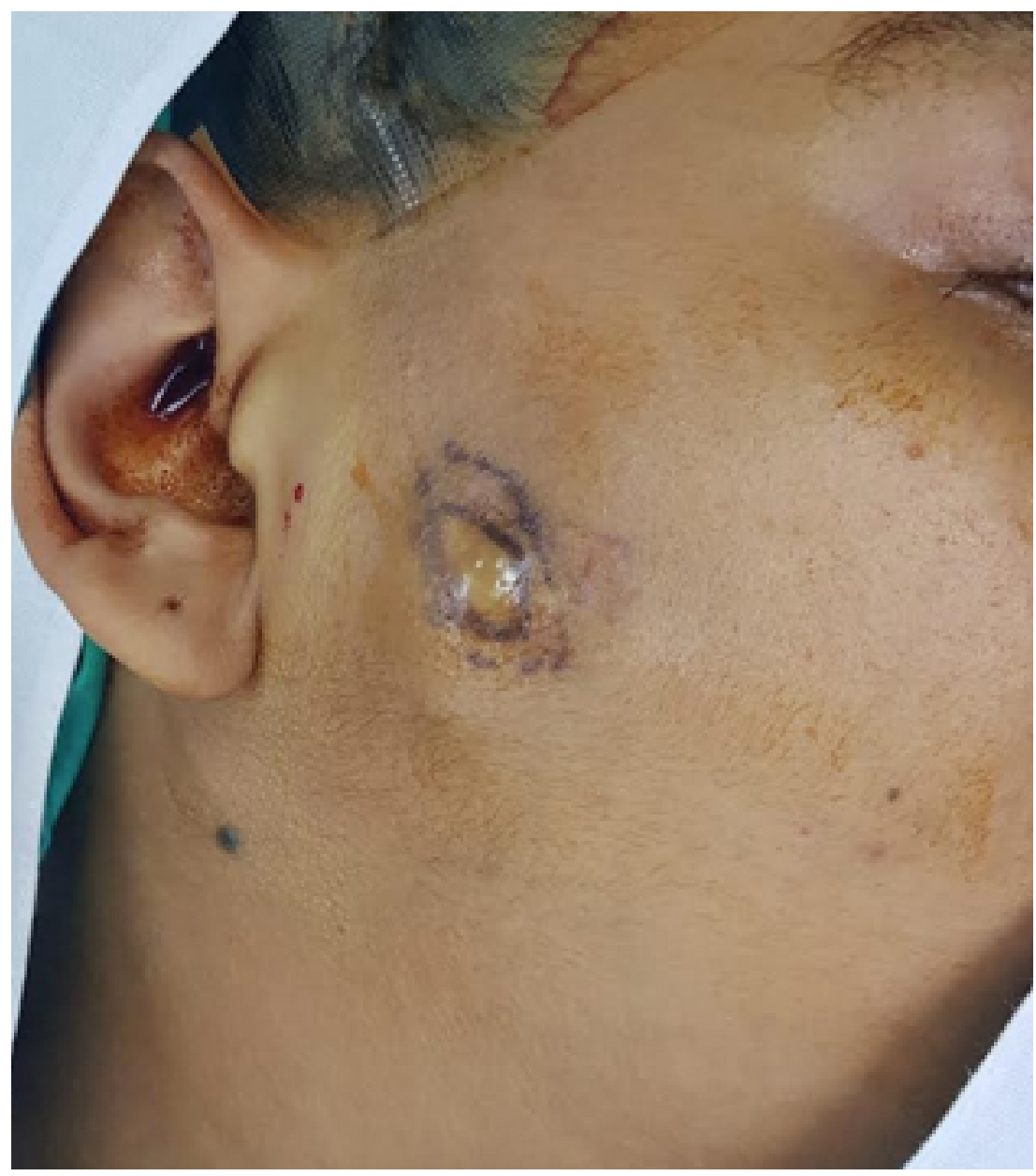

FIGURE 1: Right facial mass -AP views

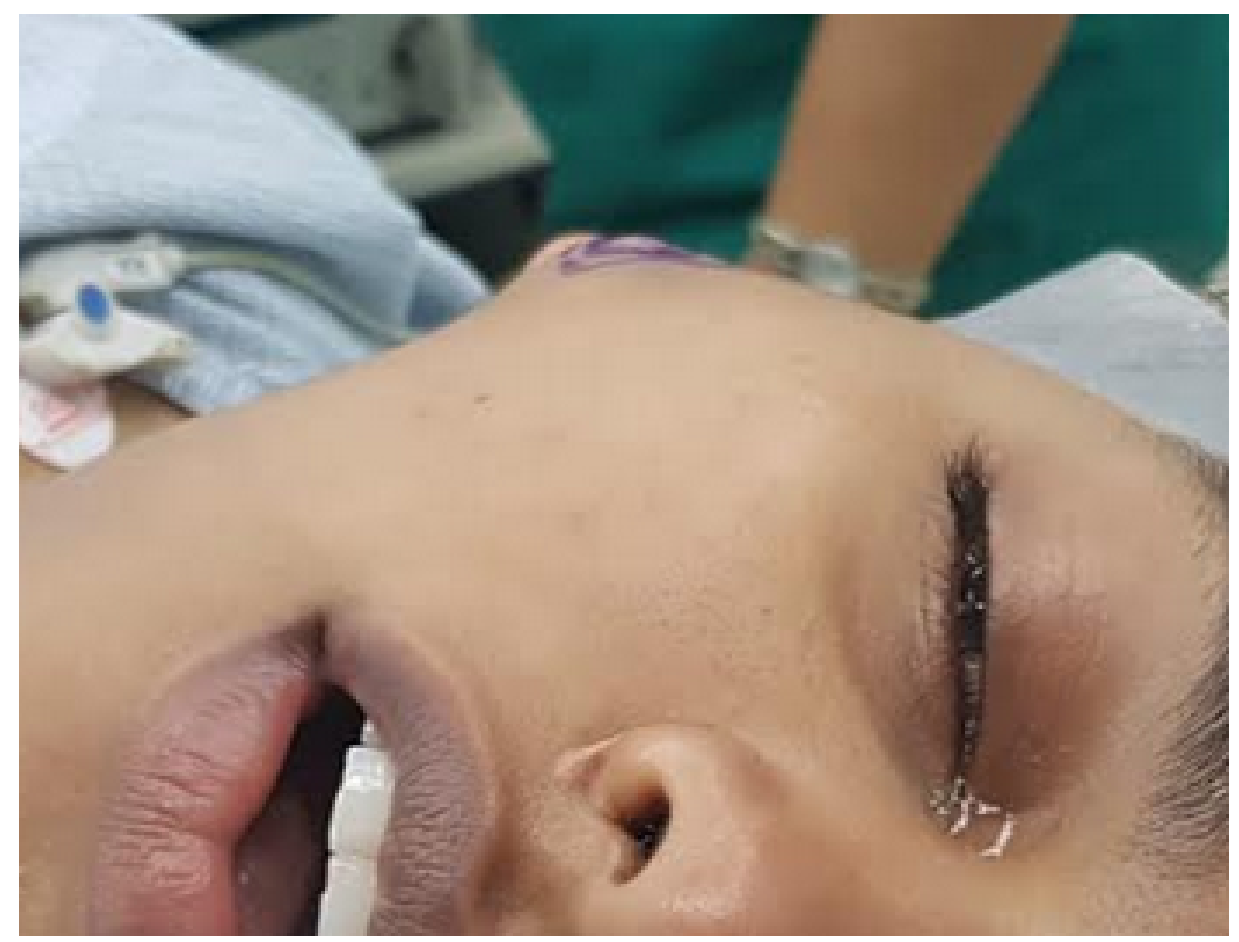

FIGURE 2: Right facial mass -AP lateral 


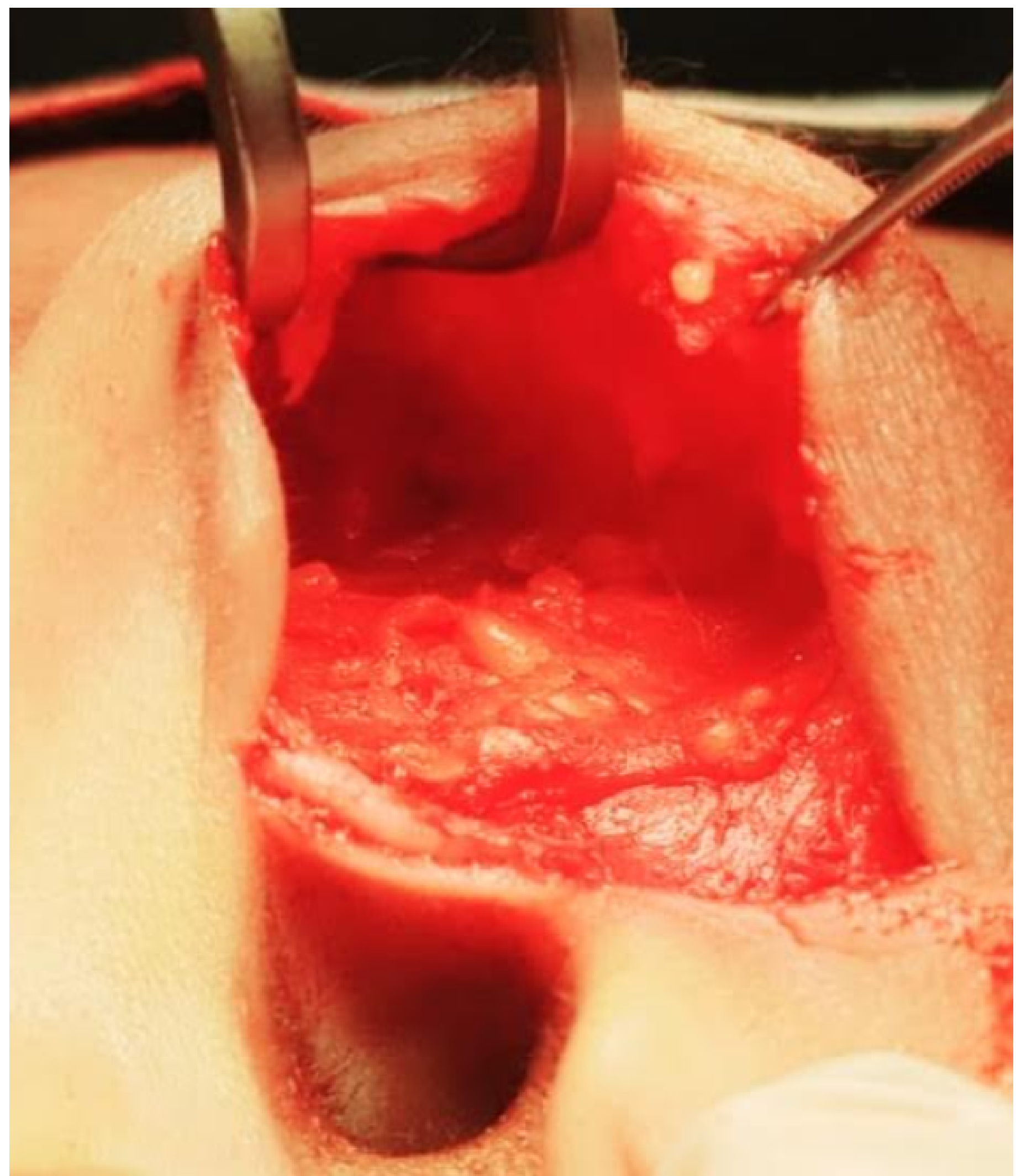

FIGURE 3: Intact right SMAS layer above the right parotid 


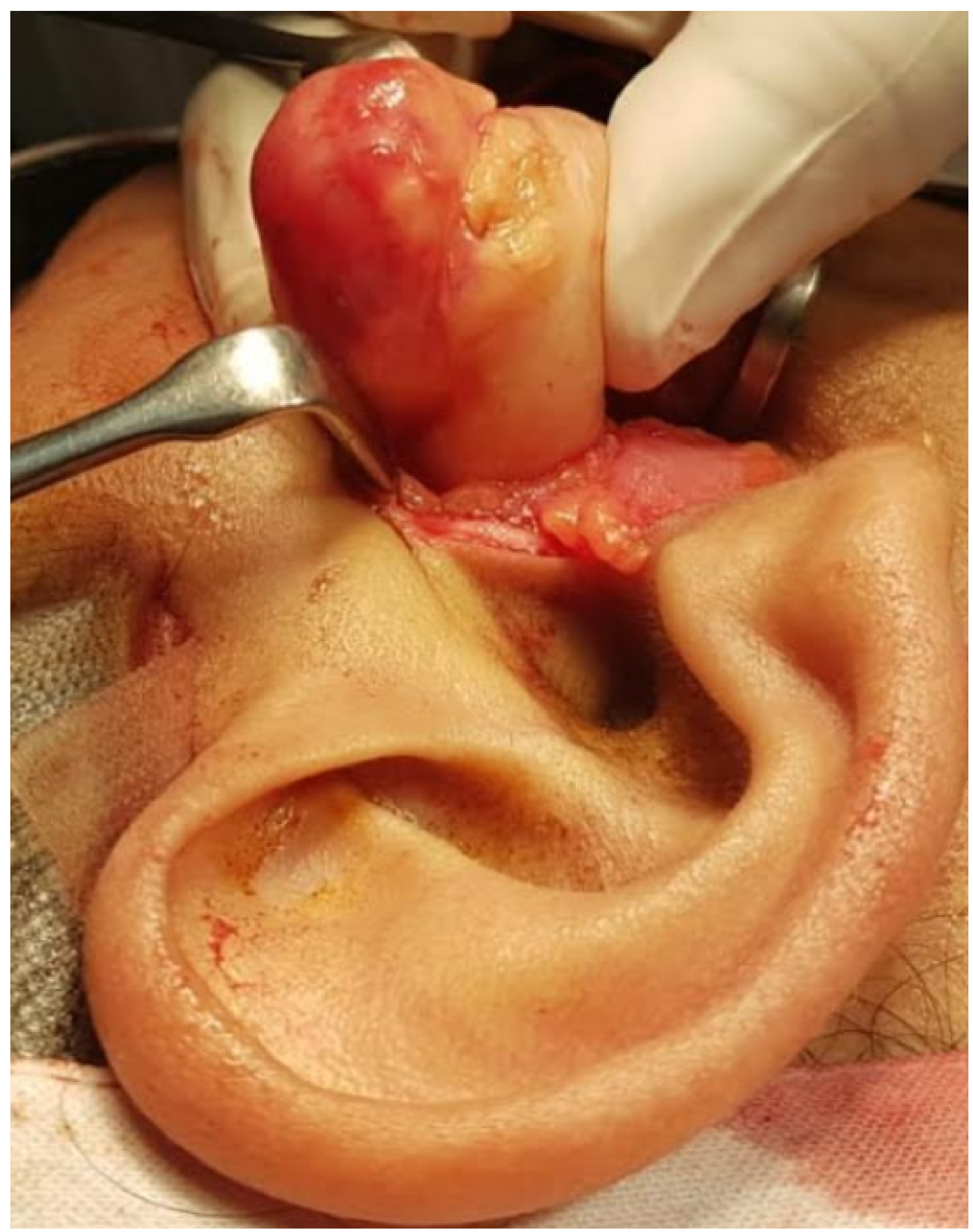

FIGURE 4: Right facial mass below the skin at the angle ofthe mandible 


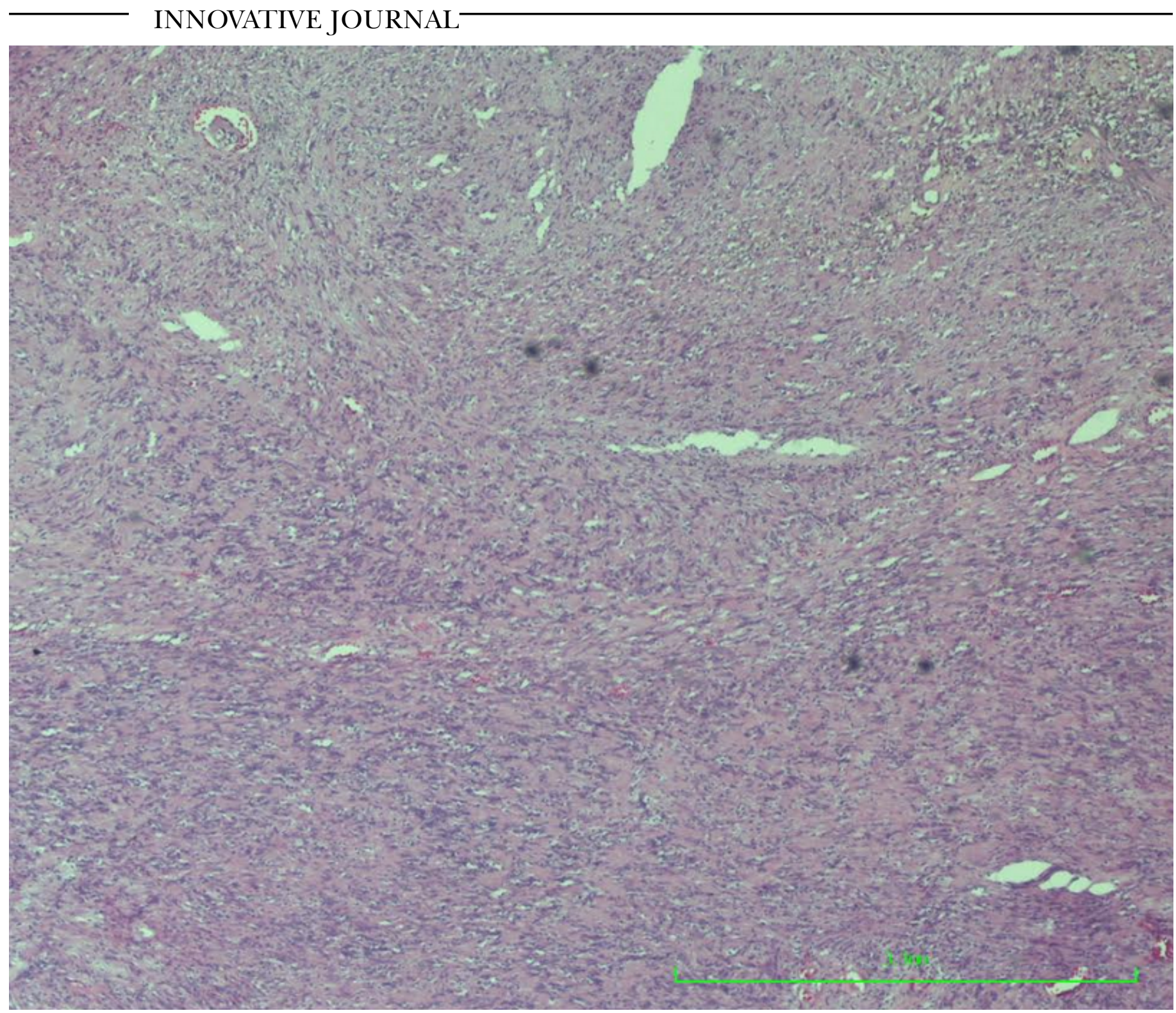

FIGURE 5: Tumour composed mostly of Antoni A patterns withverocay bodies and some Antoni B areas. H \& E stains at low $x 4$ high power $x 40$

nerves. (2, 6)The olfactory and optic cranial nerves do not have schwann cells as part of their neural coverings. (6) Genetic mutations of the NF2 gene, loss of PRKAR1A expression as well as SMARCB1 mutations have been identified in some types of schwannomas. (7) However, there is no definite aetiology. Schwannomas usually follow a benign course. (8) Malignant transformation is rare and has an annual incidence of 0.03 per 100,000 cases. (7) Patients with neurofibromatosis, prior radiation exposure or the melanotic variant are more likely to develop a malignany. $(7,9)$ Histologically, these tumours are encapsulated and can be distinguished from other tumours by the presence of Antoni type A, verocay bodies and Antoni B type areas. (10)

The vestibular schwannoma is an intracranial tumour that is present in $60 \%$ of cases. (11) It is the most common type of schwannoma. Those outside the cranium occur in $25-45 \%$ of cases. (5) In the head and neck, the parapharyngeal space is the most common location. $(12,13)$ Schwannomas can also occur within the retropharyngeal space, posterior pharyngeal wall, nose, intratemporal fossa, paranasal sinuses, tongue, larynx, epiglottis, oral cavity, submandibular gland and scalp. The cutaneous schwannoma (CS) accounts for $5 \%$ of schwannomas. These 


\section{CUTANEOUS SCHWANNOMA PRESENTING AS A PAROTID TUMOUR - A CASE REPORT AND LITERATURE REVIEW}

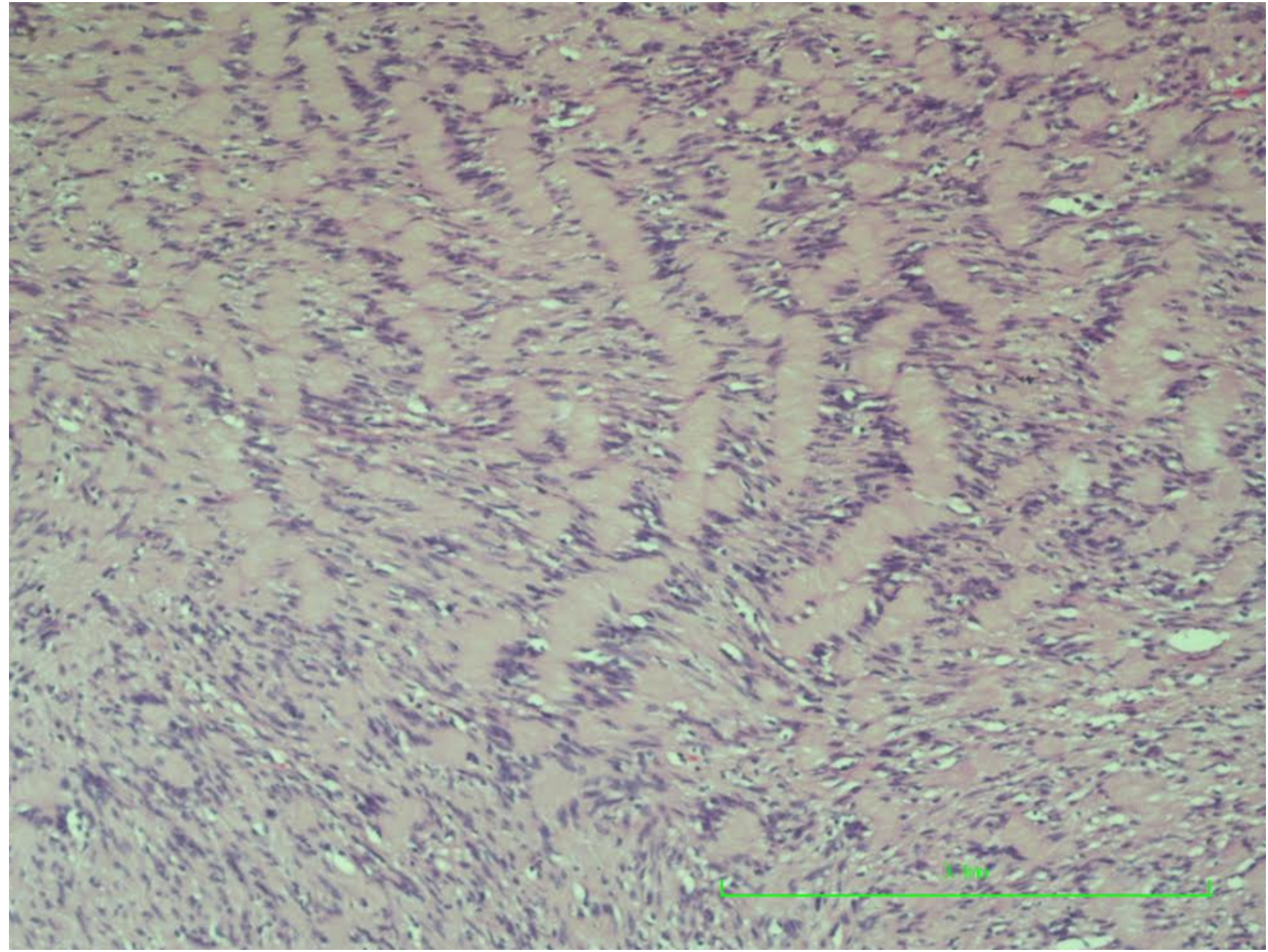

FIGURE 6: Tumour composed mostly of Antoni A patterns withverocay bodies and some Antoni B areas. H \& E stains at low $x 4$ resolution.

originate from peripheral nerves and develop within the dermis or subcutaneous tissues. (6) The tumour in our patient was located superficially over the ramus of the right jaw in the region of the parotid gland. This is not a common location for a cutaneous schwannoma. The parotid gland can also rarely develop a schwannoma and this can contribute to diagnostic confusion.

Cutaneous schwannomas have been reported to occur in young to middle aged adults. (14) However, other studies have not noted a specific age distribution. There also appears to be no predilection for ethnicity or gender. (15) As with other schwannomas, the CS grows slowly and is usually asymptomatic. It will initially appear as a lump due to its superficial location. Our patient presented with a facial lump and was concerned about cosmesis. Evaluation of the lump revealed a firm, mobile mass that was not fixed in position by jaw clenching. This supported the diagnosis of a superficial facial mass and made a parotid tumour unlikely. Some patients develop symptoms such as pain, neural deficits and structural dysfunction due to the direct pressure exerted by the schwannoma on neighboring structures. The range of differential diagnoses is wide and includes lipoma, epithelial cysts, lymph node, fibroma, desmoid tumor, leiomyoma, angioma, angiolipoma, paraganglioma, rheumatoid nodule, granular cell tumor, proliferating pilomatricoma, eccrine spiradenoma and branchial abnormalities. (4, 16, 17)

There are no hematological investigations that are pathognomonic for cutaneous schwannomas. Plain 
INNOVATIVE JOURNAL

radiographs are also not very helpful. Due to its superficial location, an ultrasound scan can give an evaluation of size, content and relation to surrounding structures. Additionally a doppler ultrasound can give information on the vascularity of the lesion. (18) If a CT scan is performed, a superficial spherical, soft-tissue mass is imaged. $(7,19)$ An MRI scan will also report a superficial oval mass which is isointense/ hypointense on T1-weighted images and hyperintense on T2-weighted images. In both CT and MRI scans, the tumour will enhance with contrast. In cases where an incisional biopsy or a trucut biopsy is taken, the histological appearance is classic. However, if there is uncertainty - immunohistochemistry can be performed. Schwannomas are positive for S100 and collagen type 4. In addition Leu-7, myelin basic protein, $\mathrm{CD} 34, \mathrm{CD} 68$, vimentin as well as collagen types 1,3 , and 4 can also stain positive. The capsule stains positive for epithelial membrane antigen. (20)In this case report, immunohistochemistry was performed on the trucut specimen and it was positive for S-100.

There are several options for the treatment of a cutaneous schwannoma. Observation is now popular as most schwannomas are benign, slow growing and asymptomatic. (3) However, patients who have tumours in the head and neck or other areas of the body that are frequently exposed may have cosmetic concerns and want more active treatment. Dysfunction of nearby structures (reduced range of movement of the neck) or pain also require another form of treatment. Surgical resection is preferred in these cases. Cutaneous schwannomas that involve terminal branches can be completely resected along with resection of the involved nerve.In our patient, the schwannoma was excised along with its terminal branch. A piece of skin where the schwannoma became attached after the trucut biopsy was performed was also removed.In larger peripheral nerves, enucleation of the schwannoma with preservation of the rest of the nerve can be performed. This is done to preserve the function of the nerve. Malignant cutaneous schwannomas also require surgical resection. In order to avoid local recurrence, a wide local excision is recommended. Adjuvant chemotherapy may improve prognosis in these patients. For benign tumours, radiotherapy is reserved for palliative cases and those who cannot undergo surgical intervention. (21)

\section{3 | CONCLUSION:}

Cutaneous schwannomas are uncommon, benign, slow-growing tumours. The aetiology is unknown and there is no age, ethnic or gender predilection. These schwannomas originate from peripheral nerves and develop within the dermis or subcutaneous tissues. CT and MRI have distinctive appearances but an ultrasound scan can provide adequate information about the tumour. Schwannomas have a characteristic histological appearance and stain positively with several stains. While observation is generally recommended, surgery is preferred when there are cosmetic concerns, pain, dysfunction of nearby structures or malignancy. Chemotherapy and radiotherapy have limited roles.

Conflicts of interest: The authors declare no conflicts of interest.

Author contribution: All authors have contributed significantly in designing and organizing to write manuscript, collecting data as well help in critical analysing the manuscript. All authors have approved the final version of this manuscript

Funding: No fund was received to publish this article.

Consent: Patient consent was obtained.

\section{REFERENCES}

1. Eroglu U, Yakar F, Zaimoglu M. Subcutaneous Schwannoma in the Head Region. Asian J Neurosurg. 2018;13(1):96-97.

2. Ritter SE, Elston DM. Cutaneous Schwannoma of the foot. Cutis. 2001;67:127-136.

3. Otávio AC, De SRP, Cunha MAMD, De JAC, Alysson P. Dedivitis Rogério Aparecido et al . Extracranial neurogenic tumors of the head and neck. Braz J otorhinolaryngol. 2015;81(6):604609. 


\section{CUTANEOUS SCHWANNOMA PRESENTING AS A PAROTID TUMOUR - A CASE REPORT AND LITERATURE REVIEW}

4. Kondo RN, Pontello R, Junior, Taguti P. Cutaneous schwannoma: an atypical presentation. An Bras Dermatol. 2017;92(3):441-442.

5. Colreavy MP, Lacy PD, Hughes J, BouchierHayes D, Brennan P, Dwyer O, et al. Head and neck schwannomas - A 10 year review. J Laryngol Otol. 2000;114:119-143.

6. Noh S, Do JE, Park JM, Jee H, Oh SH. Cutaneous schwannoma presented as a pedunculated protruding mass. Ann Dermatol. 2011;23(2):264-266.

7. Sheikh MM, De JO, Schwannoma. StatPearls [Internet. StatPearls Publishing; 2021. Available from: https://www.ncbi.nlm.nih.gov/books/ NBK562312/.

8. Shishiba T, Niimura M, Ohtsuka F, Tsuru N. Multiple cutaneous neurilemmomas as a skin manifestation of neurilemmomatosis. J Am Acad Dermatol. 1984;10:744-54.

9. Manganoni AM, Farisoglio C, Lonati A, Zorzi F, Tucci G, Pinton PG. Cutaneous epithelioid malignant schwannoma: review of the literature and case report. J Plast Reconstr Aesthet Surg. 2009;62(9):318-339.

10. Nascimento G, Nomi T, Marques R, Leiria J, Silva C, Periquito J. Ancient schwannoma of superficial peroneal nerve presenting as intermittent leg pain: A case report. Int J Surg Case Rep. 2015;6:19-22.

11. Propp JM, Mccarthy BJ, Davis FG, PrestonMartin S. Descriptive epidemiology of vestibular schwannomas. Neuro Oncol. 2006;8(1):111.

12. Hazarika P, Nayak DR, Pujary K, Rao L. Schwannoma of the nose and paranasal sinuses. Indian J Otolaryngol Head Neck Surg. 2003;55:34-42.

13. Muranjan SN, Jagasia V, Pusalkar A. Schwannoma of the cheek. Indian J Otolaryngol Head Neck Surg. 2001;53:140-141.
14. Bhandari A, Mishra P, Jain S, Sankhya N, Bapna AS. Neurofibroma of the posterior pharyngeal wall. Indian J Otolaryngol Head Neck Surg. 2000;52:161-161.

15. Sonkhya N, Luckwani A, Mishra P. Neurofibroma of the infratemporal fossa. Indian J Otolaryngol Head Neck Surg. 2001;53:142-146.

16. Ahmed S, Ahmed R, Lateef $M$, Ahmed $M$, Wani NA. Neurilem-moma (schwannoma) of the maxillary sinus. Indian J Otolaryngol Head Neck Surg. 2002;54:234-239.

17. Malone JP, Lee WJ, Levin RJ. Clinical characteristics and treatment outcome for nonvestibular schwannomas of the head and neck. Am J Otolaryngol. 2005;26:108-120.

18. Sharma DK, Sohal BS, Parmar TL, Arora H. Schwannomas of head and neck and review of literature. Indian J Otolaryngol Head Neck Surg. 2012;64:177-80.

19. Mendeszoon MJ, Cunningham N, Crockett RS, Kushner D. Schwannoma: a case report. Foot Ankle Online J. 2009;2:4-4.

20. Hood RJ, Reibel JF, Jensen ME, Levine PA. Schwannoma of the cervical sympathetic chain. The Virginia experience. Ann Otol Rhinol Laryngol. 2000;109:48-51.

21. Giangaspero F, Fratamico FC, Ceccarelli C, Brisigotti M. Malignant peripheral nerve sheath tumors and spindle cell sarcomas: An immunohistochemical analysis of multiple markers. Appl Pathol. 1989;7:134-178.

How to cite this article: J.G., I.S., C.J., M.S., C.M., A.O. Cutaneous Schwannoma Presenting as a Parotid Tumour - A Case Report and Literature Review. Innovative Journal of Medical and Health Science. 2021;1830-1838. https:// doi.org/10.52845/IJMHS/2021-11-09-21 